\title{
NUCLEAR DATA V\&V ANALYSIS FOR FUSION APPLICATIONS: INTEGRAL BENCHMARKS AND DECAY DATA
}

\author{
Olga Vilkhivskaya ${ }^{1}$, Mark Gilbert ${ }^{1}$ \\ ${ }^{1}$ UK Atomic Energy Authority, Culham Science Centre, Abingdon, OX14 3DB, UK \\ olga.vilkhivskaya@ukaea.uk, mark.gilbert@ukaea.uk
}

\begin{abstract}
A reliable estimation of the operational parameters is one of the primary concerns in the design of magnetic fusion devices such as ITER and DEMO. Methods of diagnostics and control over the critical plasma parameters determining its stability and efficiency rely on the high-energy neutron field monitoring. Extreme operational environment, such as high-energy neutron flux, electromagnetic radiation, and high temperatures might reduce the performance of the detector systems. Therefore, research and development activities in detector prototyping are carried out to address this problem. To predict the performance of the detector materials, simulations using the latest releases of the nuclear data libraries as input for the inventory codes are carried out. This paper describes the latest validation and verification (V\&V) benchmark exercise for FISPACT-II \& TENDL-2017 based on the fusion decay heat measurements performed at the Japanese FNS facility for the materials in the diagnostic components for the radiation measurements. The breakdown of decay-heat contributions from individual radionuclides have been employed to interpret the simulated results, benchmark the data against the experimental measurements, and revise the neutron-induced reactions cross-section and decay data for the associated radionuclides for the upcoming release of the TENDL-2019 nuclear data library.
\end{abstract}

KEYWORDS: fusion decay-heat, cross-section and decay data validation, gold, platinum, europium

\section{INTRODUCTION}

Recent conceptual studies with the SPECTRA-PKA code [1] of the neutronic load on the plasmaexposed first wall of the DEMO fusion power plant have shown that the per-channel analysis of the high-energy threshold reactions is required for an accurate evaluation of the damage production. The decay heat generation during operation and residual decay power assessment after power plant shutdown are also aspects requiring detailed analysis of the neutron-induced reactions in a fusion environment. The computation of the decay power performed with FISPACT-II $[2,3]$ relies on a large volume of nuclear data, including reaction cross-section and radioactive decay data. Validation and verification $(\mathrm{V} \& \mathrm{~V})$ benchmark exercises are needed to test both the code performance and the accuracy of latest releases of the nuclear databases. One of these benchmarks has been developed for neutron-irradiation applications with FISPACT-II, and relied on the fusion decay heat measurements for 74 different materials after a series of experiments performed by the Japan Atomic Energy Agency (JAEA) at their fusion neutron source (FNS) facility, [4,5]. For each irradiation case in the FNS-benchmark, FISPACT-II simulations utilise the major nuclear cross-section libraries: TENDL-2017, JEFF-3.3, ENDF/B-VIII.0, EAF2010, and IRDFF-1.05, [4,5]. 
Detailed pathway analyses with the FNS-benchmark have recently shown that while codes such as FISPACT-II generate pathway contributions from each possible reaction into the total timedependent decay heat release, the accuracy of this allocation depends entirely on the quality (and inclusion) of the cross-sections. For instance, the results discussed for several of the planned constituents of the DEMO first wall and structural materials [5] showed that either the nuclear data for certain reactions need re-evaluation or that some reaction channels to important isomeric states were completely missing, and thus should be properly embedded in the future versions of the libraries. This work presents the discussion of the FNS experiment results for the elements relevant in the radiation and spectroscopy measurements.

\section{ANALYSIS OF THE FNS EXPERIMENTAL RESULTS ON DECAY HEAT FOR THE MATERIALS RELEVANT TO THE DIAGNOSTICS APPLICATIONS IN FUSION}

Diagnostics currently being designed and tested in simulated fusion environment include detectors based on the foil activation technique, [6-8]. The following sections provide a revision of the FISPACT-II \& TENDL-2017 simulation results for the FNS decay-heat experiment for gold, platinum, and europium after five-minute irradiation. In these materials, the primary contributions to the total decay-heat are attributed to the metastable isomers that are produced via the $(n, 2 n)$ reaction channel. Currently, the pathways to these important isomeric states for gold and europium are properly embedded only in TENDL and EAF2010 reaction cross-section data libraries, [4], which ensures that the identification of the isomer is identical in the decay data file $d e c .2012$ used in the simulations. The isomeric branching ratios in $(n, 2 n)$ reaction channel is of particular concern.

\subsection{Gold}

The main-vessel and divertor bolometry in JET [9] relies on miniature metal foil detectors comprised of a gold-absorbing layer on a thin mica substrate and a gold meander on the rear side. Therefore, the accuracy of the radiation measurement interpretation depends on taking into account the quality of the evaluated cross-section data utilised for estimating the high-energy neutroninduced reactions in gold. Figure 1 shows that TENDL-2017 decay-heat simulation results for gold overestimate the experimental values by about $30 \%$.

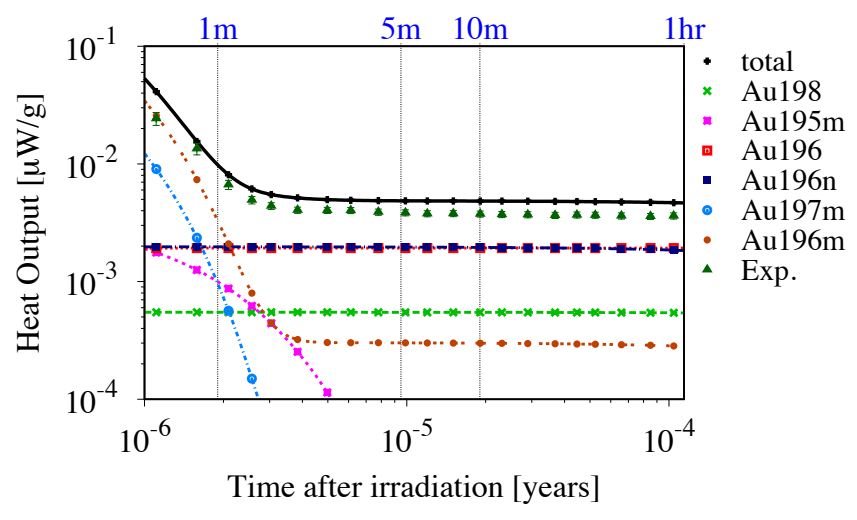

Figure 1: FISPACT-II\&TENDL-2017 simulation results for the decay-heat experiment for gold in the FNS benchmark, [4]. 
Incorrect branching ratios for $(\mathrm{n}, 2 \mathrm{n})$ to ${ }^{196} \mathrm{Au},{ }^{196 m} \mathrm{Au}$, and ${ }^{196 n} \mathrm{Au}$ in TENDL-2017 could be the source of overestimation with that library, which can be seen in Figure 2. Taking into consideration the evaluated cross-section for the ${ }^{197} \mathrm{Au}(\mathrm{n}, 2 \mathrm{n}){ }^{196} \mathrm{Au}$ in the IRDFF-1.05 [10] dosimetry library, as well as the available EXFOR [11] experimental data suggests that the branching ratio for the ${ }^{196} \mathrm{Au}$ and ${ }^{196 m} \mathrm{Au}$ (see Table 1) in TENDL needs adjustment to balance the production of these nuclides taking into account their isomeric transitions (IT). Table 1 provides the details of principal reaction product pathways, branching ratios for the decay modes, half lives and associated gamma lines: gamma energy $E_{\gamma}$ and intensity $I$.

In addition to the recommended and frequently utilised reaction pathways for dosimetry [10] and activation [7] assessment for fusion and fission applications, measurement of short-lived product nuclides is important for producing rapid diagnostics for the neutron energy spectra parameters. For instance, the experimental results obtained at the ASP $14 \mathrm{MeV}$ neutron irradiation facility [6] for ${ }^{197} \mathrm{Au}\left(\mathrm{n}, \mathrm{n}^{\prime}\right)^{197 m} \mathrm{Au}$ have shown that it was possible to track the derived activity over time. Thus this reaction could be utilised in rapid diagnostics and would benefit from additional integral crosssection measurements in the 5-15 MeV region, see Figure 2.
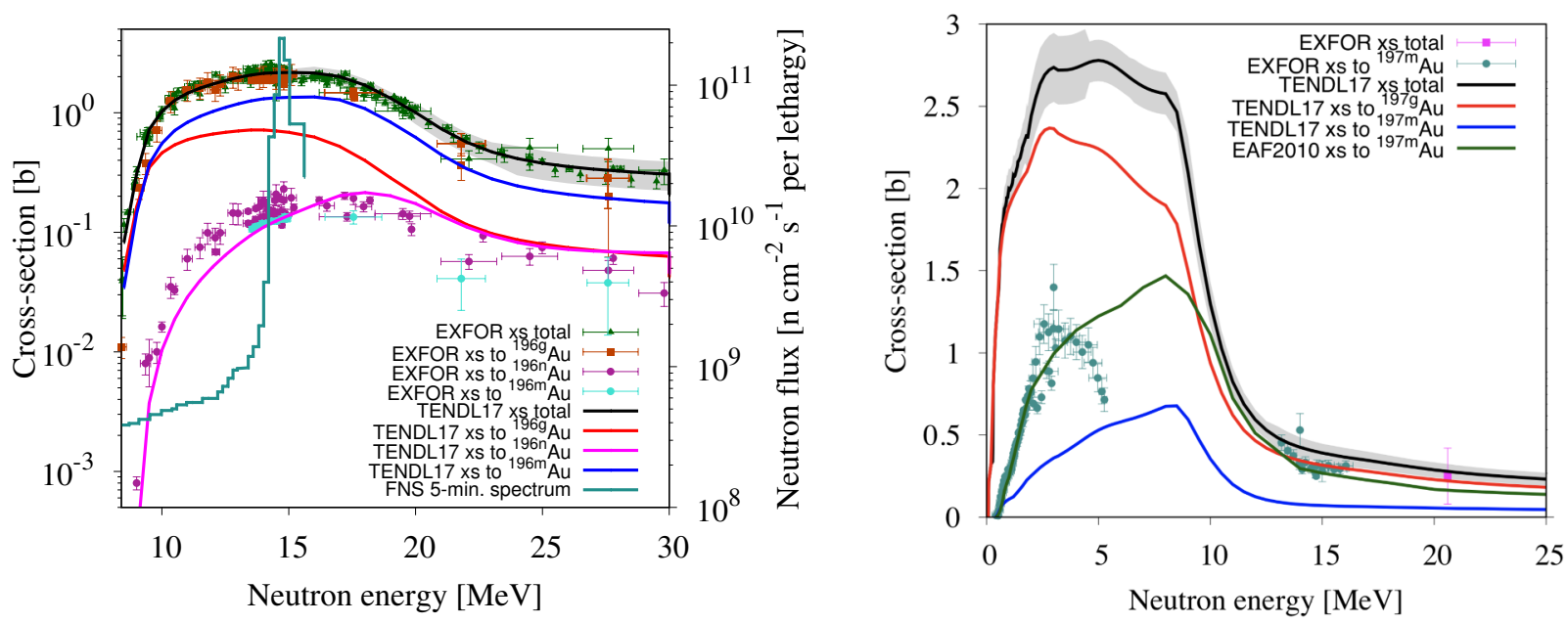

Figure 2: Comparison of evaluated cross-section for ${ }^{197} \mathrm{Au}(\mathrm{n}, 2 \mathrm{n})$ reaction channel (left) and ${ }^{197} \mathrm{Au}\left(\mathbf{n}, \mathbf{n}^{\prime}\right)$ reaction channel (right) along with the EXFOR experimental data.

Table 1: Details of the investigated reaction pathways for gold, [12]

\begin{tabular}{|c|c|c|c|c|}
\hline Reaction & Product & Decay mode & $T_{1 / 2}$ & Principal $E_{\gamma}, \operatorname{keV}(I, \%)$ \\
\hline \multirow{3}{*}{${ }^{197} \mathrm{Au}(\mathrm{n}, 2 \mathrm{n})$} & ${ }^{196 m} \mathrm{Au}$ & IT & $8.10 \mathrm{~s}$ & $84.66(0.305)$ \\
\hline & ${ }^{196 n} \mathrm{Au}$ & $\mathrm{IT}_{\mathrm{m}}: 97.58 \% ; \mathrm{IT}_{\mathrm{g}}: 2.42 \%$ & $9.60 \mathrm{~h}$ & $148(42.5)$ \\
\hline & ${ }^{196} \mathrm{Au}$ & $\beta^{+}: 92.8 \% ; \beta^{-}: 7.2 \%$ & $6.1669 \mathrm{~d}$ & $355.73(87.0) ; 426.10(6.6)$ \\
\hline${ }^{197} \mathrm{Au}\left(\mathrm{n}, \mathrm{n}^{\prime}\right)$ & ${ }^{197 m} \mathrm{Au}$ & IT & $7.74 \mathrm{~s}$ & $279.0(70.8)$ \\
\hline
\end{tabular}

\subsection{Platinum}

Apart from being one of the minor transmutation products of tungsten in the fusion environment, platinum is planned to be an important component of bolometers for the radiation measurements 
in ITER, $[9,13]$, and is also considered for DEMO, [14]. In the ITER environment, Pt-based bolometers are expected to withstand high thermal loads due to high neutron fluence and radiation damage up to $0.3 \mathrm{dpa}$ (tested up to $0.1 \mathrm{dpa}$ ), $[3,14]$. The proposed scheme for DEMO implies that bolometers are planned to be placed at approximately $10 \mathrm{~m}$ from the first wall, in direct view of plasma, thus the nuclear heating must be taken into account for accurate measurements. In case of Au-based bolometers, about $10 \%$ of Au is expected to transmute into $\mathrm{Hg}$ during the ITER lifetime due to the high neutron capture cross-section, [9], which could particularly affect the temperature coefficient of resistance (TCR). Therefore, the design of Pt-based (absorber and resistor) detectors relies on its the higher melting temperature (stability of thin absorber layers), transmutation to stable Pt isotopes, and linear function of the TCR.

Analysis of the decay-heat simulation results with TENDL-2107 library for platinum suggests that the only nuclide predicted to contribute significantly to the decay-heat is ${ }^{197 m} \mathrm{Pt}\left(\mathrm{T}_{1 / 2}=1.59 \mathrm{~h}\right)$ at all cooling times, see figure 3 , and the discrepancy between the simulations and experiment must be attributed to incorrect production rates of that nuclide (around a $20 \%$ over-prediction in the decayheat from $\left.{ }^{197 m} \mathrm{Pt}\right)$. The EXFOR cross-section data for the entire ${ }^{198} \mathrm{Pt}(\mathrm{n}, 2 \mathrm{n})$ channel in Figure 3 reveals that the current evaluated cross section for ${ }^{197 m} \mathrm{Pt}$ in TENDL-2017 in the 13-15 MeV energy region could be biased by the high data points at $15-17 \mathrm{MeV}$, and the large scatter in the total cross-section points. Only a few available data points correspond to the ground state of ${ }^{197} \mathrm{Pt}$ which suggests that future data acquisition in the $9-15 \mathrm{MeV}$ energy region would be particularly beneficial for this element for the verification of the branching ratio of that channel.

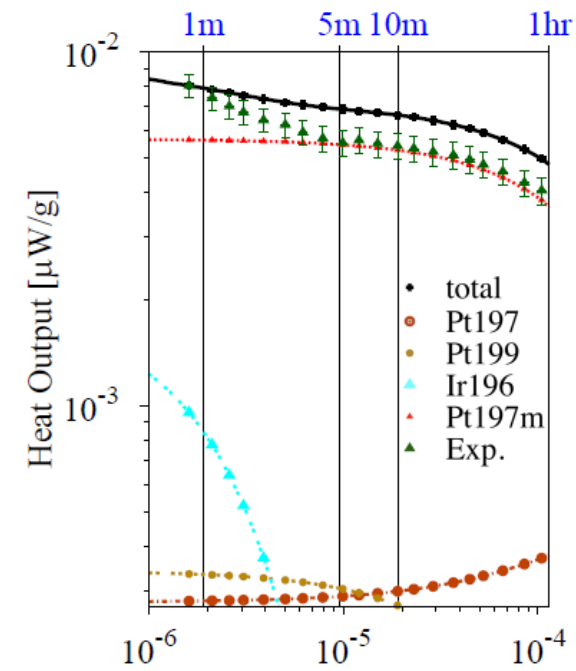

Time after irradiation [years]

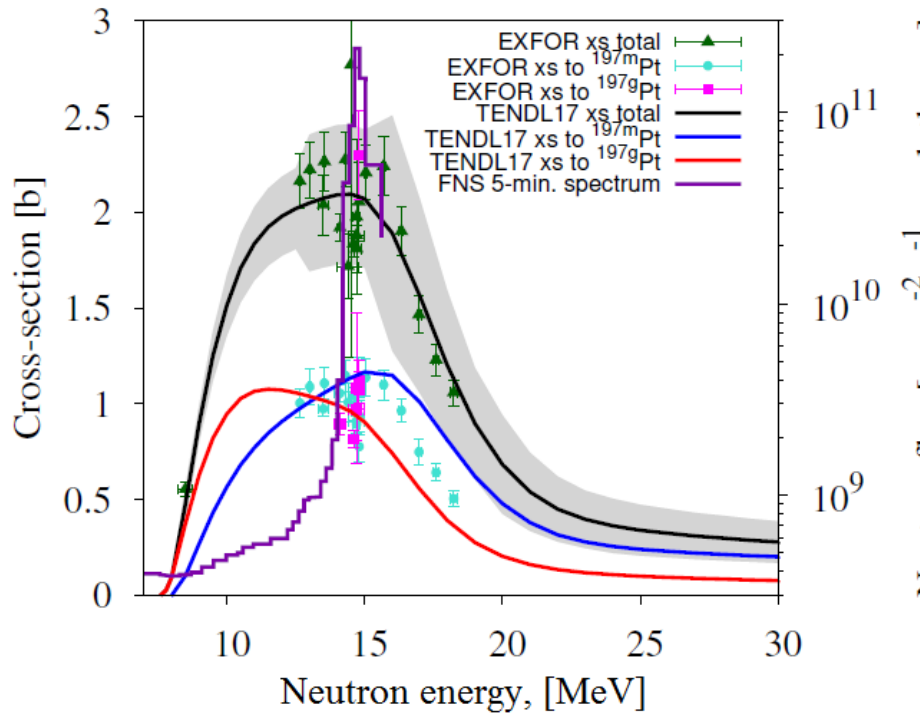

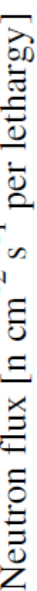

Figure 3: FISPACT-II\&TENDL-2017 simulation results for the decay-heat experiment for platinum (left) in the FNS benchmark, [4], and TENDL-2017 cross-section for ${ }^{198} \mathbf{P t}(\mathbf{n}, 2 \mathrm{n})$ reaction channel along with the EXFOR experimental data (right).

\subsection{Europium}

Europium-doped compound scintillators for gamma-ray spectroscopy applications rely on the radioluminescence characteristics of europium. Recent investigation of thermoluminescence radiation 
dosimetry characteristics of europium-doped materials have shown promising results, as well as the experiments at the FNS facility (see referenced works by Maekawa et al. in [5]). The FNS experiment results for europium shown in Figure 4 (irradiated in the form of $\mathrm{Eu}_{2} \mathrm{O}_{3}$ sandwiched between plastic tape) reveal an upward trend in the decay-heat measurements at short cooling times (less than 5 minutes), which is not reproduced in the simulations with TENDL-2017, [4]. As discussed in [4], either the decay heat contribution due to the plastic tape is overestimated or there are some missing isomeric states in the simulations that could explain the disagreement with the experiment results. However, review of the EXFOR cross-section data shown in Figure 5 and recent findings by the authors in [15] suggest a re-evaluation and adjustment of the branching ratio for the ${ }^{153} \mathrm{Eu}(\mathrm{n}, 2 \mathrm{n})$ reaction channel for future TENDL library release: derived corresponding cross-sections at 13.5, 14.1 and $14.8 \mathrm{MeV}$ agree with the majority of EXFOR data for each product nuclide in this reaction (taking into account the experimental uncertainties). Re-evaluation of the cross-section curves with respect the majority of the experimental data points would result in a lower production rate of the shorter-lived isomers ${ }^{152 m} \mathrm{Eu}$ and ${ }^{152 n} \mathrm{Eu}$ that dominate the decay heat after 5 minutes of cooling time.

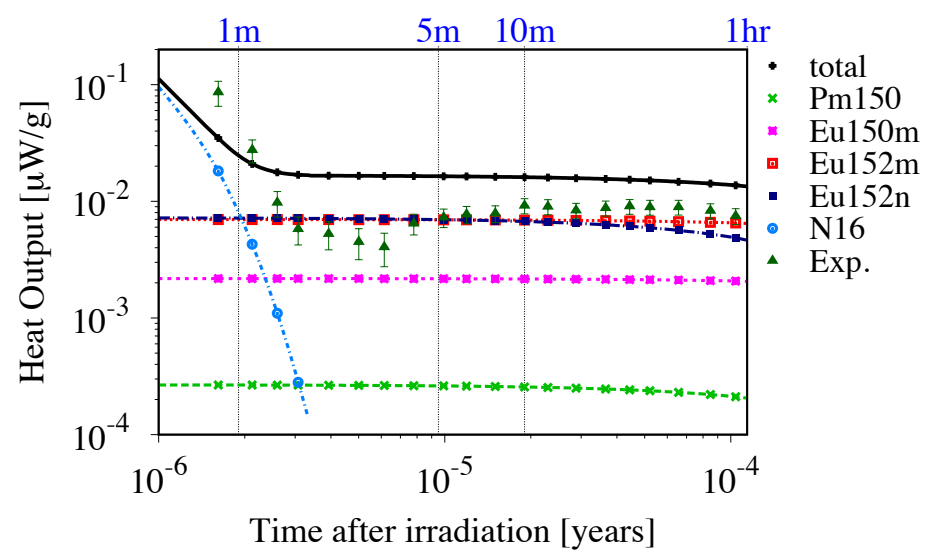

\section{Figure 4: FISPACT-II\&TENDL-2017 simulation results for the decay-heat experiment for europium oxide in the FNS benchmark, [4].}

It should also be noted that ${ }^{16} \mathrm{~N}$ dominates the decay-heat during the first minutes of the cooling period for europium oxide, see Figure 4, however, the simulation results under-predict the experiment. This under-prediction of ${ }^{16} \mathrm{~N}$ contributions at short timescales was noted for all irradiated oxides described in the report [4], and would be discussed in more detail by L.W. Packer et al. in their contribution to this conference [16].

The neutron-induced reaction cross-sections for Eu have been evaluated and incorporated into major nuclear data libraries. However, available EXFOR data revealed only a limited amount of data points associated with the isomeric states of the product nuclides in the ${ }^{153} \mathrm{Eu}(\mathrm{n}, 2 \mathrm{n})$ reaction channel, and particularly only one data point for the ground state ${ }^{152} \mathrm{Eu}$, see Figure 5 . Recent study [15] of the (n,2n) reaction channel in natural europium $\left({ }^{151} \mathrm{Eu} 47.81 \%\right.$ and $\left.{ }^{153} \mathrm{Eu} 52.19 \%\right)$ suggests that a complex $\gamma$-ray spectrum associated with the ground state of the product nuclei and their long half-life (see Table 2) could be contributing to the observed discrepancies in the cross-section experimental data. 
Table 2: Details of the investigated reaction pathways for europium, $[17,18]$

\begin{tabular}{|c|c|c|c|c|}
\hline Reaction & Product & Decay mode & $\mathrm{T}_{\mathbf{1} / \mathbf{2}}$ & Principal $E_{\gamma}, \mathrm{keV}(I, \%)$ \\
\hline \multirow{2}{*}{${ }^{153} \mathrm{Eu}(\mathrm{n}, 2 \mathrm{n})$} & ${ }^{152 m} \mathrm{Eu}$ & $\beta^{+}: 27.0 \% ; \beta^{-}: 73.0 \%$ & $9.3116 \mathrm{~h}$ & $841.63(14.2) ; 344.29(2.4)$ \\
& ${ }^{152 n} \mathrm{Eu}$ & $\mathrm{IT}$ & $96 \mathrm{~m}$ & $89.85(69.7)$ \\
& ${ }^{152} \mathrm{Eu}$ & $\beta^{+}: 72.1 \% ; \beta^{-}: 27.9 \%$ & $13.517 \mathrm{y}$ & $121.78(28.53) ; 344.28(26.59)$ \\
\hline${ }^{151} \mathrm{Eu}(\mathrm{n}, 2 \mathrm{n})$ & ${ }^{150 m} \mathrm{Eu}$ & $\beta^{+}: 11.0 \% ; \beta^{-}: 89.0 \%$ & $12.80 \mathrm{~h}$ & $333.9(4.0)$ \\
& ${ }^{150} \mathrm{Eu}$ & $\beta^{+}$ & $36.9 \mathrm{y}$ & $333.97(95.16)$ \\
\hline
\end{tabular}
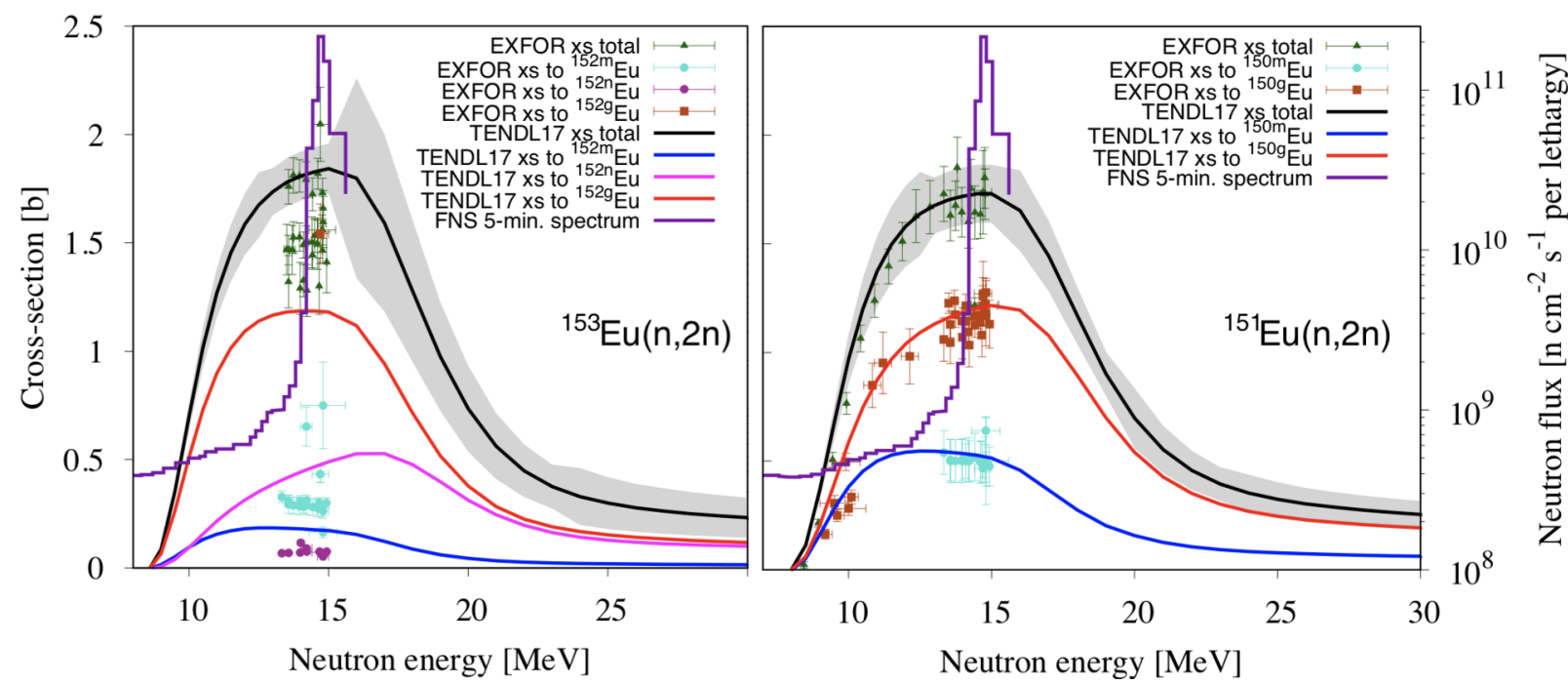

Figure 5: TENDL-2017 evaluated cross-section for ${ }^{153} \operatorname{Eu}(\mathbf{n}, 2 \mathrm{2n})$ and ${ }^{151} \mathrm{Eu}(\mathbf{n}, 2 \mathrm{2n})$ reaction channels along with the EXFOR experimental data.

\section{RESULTS AND ANALYSIS OF THE DECAY DATA}

The decay library currently used by FISPACT-II is almost a decade old and needs to be updated and re-validated with more recent experimental data. This 3875-nuclide decay library dec_2012 was assembled from eaf_dec_2010 complemented with all of JEFF-3.1.1 (3331) files, ENDF/B-VII (78), UKPADD (90, from versions 6.5-6.10) and a handful of the files from the older versions of other decay libraries, $[4,12]$. The dec_2012 decay library was used by default in the simulations with TENDL-2017 presented above. However, ENDF/B-VIII.0 [19] and JEFF-3.3 [20] have their own dedicated decay libraries. The current version of the JEFF-3.3 decay data library has files for 3851 nuclides with consistent source and new evaluations from NUBASE basic nuclide properties database. The decay data sublibrary in ENDF/B-VIII.0 release (3820 nuclides) has been reviewed and updated, including, for instance, the beta intensities following $\beta^{-}$-decay. The intermediate review results for the source files in the dec_2012 suggest the files from the latest decay library releases: JEFF-3.3 (3683), UKPADD-6.12 (90), and ENDF/B-VIII.0 (78), as well as reviewing outdated files (24) and adding the files for the nuclides considered in ENDF/B-VIII.0 (19) that 
include important isomers. Additional nuclides from JENDL Decay Data File (2015) should be considered.

Review of the decay files included in $d e c 2012$ for the product nuclides in the discussed reaction channels for gold, platinum and europium revealed that the original data sources in the JEFF-3.3 decay library files remained unchanged compared to those in JEFF-3.3.1. In particular, the decay files for ${ }^{197 m} \mathrm{Au}$ and ${ }^{197 m} \mathrm{Pt}$ highlight the fact that further $\gamma$-ray studies are required to confirm the validity of the proposed isomeric transition (IT) decay scheme, and particularly to quantify the absolute $\gamma$-ray emission probabilities. ${ }^{197 m} \mathrm{Pt}$ undergoes IT decay to ${ }^{197} \mathrm{Pt}$, and $\beta$-decay to ${ }^{197 m} \mathrm{Au}$, however only two $\gamma$-rays are directly associated with the decay scheme of ${ }^{197 m} \mathrm{Pt}$ (53.10 and 346.5 $\mathrm{keV} \gamma$-ray transitions from the IT decay mode). The other $\gamma$-rays are identified with the subsequent IT decay of ${ }^{197 m} \mathrm{Au}$, and have been incorporated into the data file of this daughter radionuclide. The decay scheme of ${ }^{197 m} \mathrm{Pt}$ was derived from the estimated branching fractions: IT branching fraction of $96.7 \%$, and $\beta$-decay branching fraction of $3.34 \%$, with the 53.10 and $346.5 \mathrm{keV} \gamma$-rays representing a two-transition cascade from the $399.59 \mathrm{keV}$ metastable state. Therefore, further gamma-ray measurements are still needed to confirm the absolute emission probabilities of the two $\gamma$-rays, [21].

The results in the most recent work [15] that investigated the complexity of the characteristic $\gamma$-rays attributed to the isomeric/ground state products in ${ }^{153} \mathrm{Eu}(\mathrm{n}, 2 \mathrm{n})$ reaction have highlighted the issue with the evaluated data for this reaction channel. However, the authors in this work also draw attention to the half-life of 12.4 years that was used by Qaim [15] to determine the only one data point for the cross-section resulting in the ground state ${ }^{152} \mathrm{Eu}$ (see Figure 5). More recently reported updated half-life figures presented in Table 2 are in agreement with the data in dec_2012 therefore favouring the suggestion for re-evaluation of the branching ratio for ${ }^{153} \mathrm{Eu}(\mathrm{n}, 2 \mathrm{n})$ evaluated cross-section in future TENDL releases.

\section{CONCLUSIONS}

The results of the present work suggest that adjustment (re-evaluation) of the cross-sections and/or branching ratios for important $(n, 2 n)$ reaction pathways to the isomeric states is needed in the next release of the TENDL library for the materials relevant in bolometry and gamma-ray spectroscopy applications in fusion. The analysis provided for the dominant reaction pathways, which were identified in the decay-heat benchmarking exercise for FISPACT-II \& TENDL-2017 for gold, platinum and europium, revealed the complexity of the decay scheme in these materials after a five-minute irradiation in the JAEA FNS neutron spectrum. The suggested revision of both the evaluated

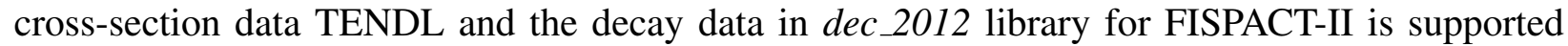
by the available experimental data in EXFOR. However, additional integral experiments for these materials in fusion-relevant spectrum would be beneficial for the validation of the evaluated reaction cross-sections and improvement of the agreement between the experimental results and the FISPACT-II decay heat simulations.

The ongoing project for the updated decay library is primarily focussed on the decay files for the radionuclides identified as critical/important for DEMO activation, dose-rate, decay heat, and waste analyses. The review of the current status of decay data for fusion activation applications will be performed in more detail to identify any discrepant data (based on experimental validation) and to also assess if there are any available updates (from published experimental data) for fusion-critical 
radionuclides. Further works will primarily include (but would not be limited to) the constituents of the candidate materials for the typical DEMO design, [3,5]: tungsten and its transmutation products (first wall), EUROFER and SS316 (structural materials), and material compositions in four different combined cooling and tritium breeding concepts.

\section{ACKNOWLEDGEMENTS}

This work has been carried out within the framework of the EUROfusion Consortium and has received funding from the Euratom research and training programme 2014-2018 under grant agreement No.633053 and from the RCUK Energy Programme (grant number EP/T012250/1). The views and opinions expressed herein do not necessarily reflect those of the European Commission. To obtain further information on the data and models underlying this paper please contact PublicationsManager@ukaea.uk.

\section{REFERENCES}

[1] M.R. Gilbert, J.-Ch. Sublet. "Differential dpa calculations with SPECTRA-PKA.” Journal of Nuclear Materials, (504), pp. 101-108 (2018).

[2] J.-Ch. Sublet et al. "FISPACT-II: an advanced simulation system for activation, transmutation and material modelling." Nucl Data Sheets, volume 139, pp. 77-137 (2017).

[3] G. Federici et al. "European DEMO design strategy and consequences for materials." $\mathrm{Nu}$ clear Fusion, volume 57, p. 092002 (2017).

[4] "Fusion decay heat validation, FISPACT-II \& TENDL-2017, EAF2010, ENDF/B-VIII.0, JEFF-3.3, and IRDFF-1.05 nuclear data libraries. CCFE-R(18)002.” (2018). URL https: //fispact.ukaea.uk/documentation-2/reports/.

[5] M.R. Gilbert, J.-Ch. Sublet. "Experimental decay-heat simulation-benchmark for $14 \mathrm{MeV}$ neutrons \& complex inventory analysis with FISPACT-II." Nuclear Fusion, volume 59, p. 086045 (2019).

[6] L.W. Packer et al. "UK fusion technology experimental activities at the ASP $14 \mathrm{MeV}$ neutron irradiation facility." Fusion Engineering and Design, volume 87, pp. 662-666 (2012).

[7] L.W. Packer et al. "Activation of ITER materials in JET: nuclear characterisation experiments for the long-term irradiation station." Nuclear fusion, volume 58, p. 096013 (2018).

[8] C.R. Nobs et al. "Neutron spectrum unfolding for the development of a novel neutron detector for fusion." Fusion Engineering and Design, volume 146, Part B, pp. 2658-2662 (2019).

[9] H. Meister et al. "Current status of the design of the ITER bolometer diagnostic." Fusion engineering and design, volume 120, pp. 21-26 (2017).

[10] R. Capote et al. "Updating and Extending the IRDF-2002 Dosimetry Library." ASTM International, volume 9 (2012).

[11] "Experimental Nuclear Reaction Data (EXFOR).” (2019). URL https://www-nds.iaea.org/ exfor/.

[12] "The European Activation File: EAF-2010 Decay Data Library. CCFE-R(10)02." (2010). URL http://www.ccfe.ac.uk/assets/Documents/CCFE-R(10)02.pdf. 
[13] Suarez A. et al. "Neutronic analysis for bolometers in ITER." Fusion Engineering and Design, volume 88, pp. 1395-1399 (2013).

[14] F.P. Orsitto et al. "Diagnostics and control for the steady state and pulsed tokamak DEMO." Nuclear fusion, volume 56, p. 026009 (2016).

[15] J. Luo et al. "Activation cross section and isomeric cross section ratios for the (n,2n) reaction on ${ }^{153}$ Eu." Physical Review C, volume 96, p. 044617 (2017).

[16] L.W. Packer et al. "A comparison of oxide decay heat simulations and nuclear data libraries." These proccedings (2020).

[17] M.J. Martin et al. "Nuclear Data Sheets for A = 152." Nucl Data Sheets, volume 114, pp. 1497-1847 (2013).

[18] S.K. Basu, A.A. Sonzogni. "Nuclear Data Sheets for A=150." Nucl Data Sheets, volume 114, pp. 435-660 (2013).

[19] D.A. Brown et al. "ENDF/B-VIII.0: The 8th Major Release of the Nuclear Reaction Data Library with CIELO-project Cross Sections, New Standards and Thermal Scattering Data." Nuclear Data Sheets, (148), pp. 1-142 (2018).

[20] O. Cabellos et al. "Benchmarking and validation activities within JEFF project." EPJ Web of Conferences, (146), p. 06004 (2017).

[21] H. Xiaolong, Zh. Chunmei. "Nuclear Data Sheets for A=197.” Nucl Data Sheets, volume 104, pp. 283-426 (2005). 